ISSN: 2644-1403

\title{
Is There A Neck-Shoulder Syndrome?
}

\author{
Jeremy Simon ${ }^{2}$, Jeffrey Gehret ${ }^{2}$, Garett Helber ${ }^{2}$, Christopher Mehallo $^{3}$, Michael Mehnert ${ }^{2}$ and Robin Raju ${ }^{1 *}$ \\ ${ }^{1}$ Department of Orthopedics and Rehabilitation, Yale School of Medicine, USA
}

${ }^{2}$ Department of Physical Medicine and Rehabilitation, Thomas Jefferson University Hospital, USA

${ }^{3}$ Department of Sports Medicine, Thomas Jefferson University Hospital, USA

*Corresponding author: Robin Raju, Clinical Assistant Professor, Interventional Spine and Musculoskeletal Medicine, Physical Medicine and Rehabilitation, Department of Orthopedics and Rehabilitation, Yale School of Medicine/Yale New Haven Hospital, USA

\begin{abstract}
Concomitant presentation of neck and shoulder pain is a common clinical scenario which can present a significant diagnostic and therapeutic dilemma. Neck and shoulder pain presentations can be separated into four different categories: Primary neck pathology with referred pain to the shoulder, primary shoulder pathology with referred pain to the neck, primary neck and primary shoulder pathology, and primary neck pathology resulting in secondary shoulder pathology. Primary neck pathology resulting in secondary shoulder pathology is mechanically plausible but not proven. Authors are proposing this scenario to be described as "neck-shoulder syndrome." For instance, C5 and/or C6 cervical radiculopathy can result in rotator cuff, deltoid, biceps and scapular muscle weakness as these nerve roots innervate the shoulder girdle musculature which in turn could produce shoulder/scapular muscle imbalance resulting in shoulder impingement signs. A patient may present with features of both cervical radiculopathy and shoulder impingement syndrome in this scenario. At this time there are no agreed clinical criteria for a diagnosis of "neck-shoulder syndrome." As with any other syndrome, management differences can only be well studied once the entity has been properly defined. In this article, authors set out to summarize how to best approach patients presenting with both neck and shoulder pain while describing features of proposed "neck-shoulder syndrome." It is paramount to take a comprehensive and holistic approach towards patients presenting with concomitant neck and shoulder pain as the symptoms may not always represent isolated entities.
\end{abstract}

Keywords: Neck and shoulder pain; Neck-shoulder syndrome; Pain treatment; Differential diagnosis of neck and shoulder pain

\section{Introduction}

Co-existent neck and shoulder pain has been described in limited fashion in the literature as a unique diagnosis, but the concomitant presentation of neck and shoulder pain is a common scenario in primary care and orthopedic offices [1,2]. Gorski et al described "shoulder impingement syndrome" where patients presented with neck pain secondary to rotator cuff tendinopathy [1]. Compere et al described a "neck, shoulder, and arm syndrome" which primarily referred to neuropathic pain in the neck, shoulder and arm resulting from a brachial plexus lesion [2]. When patients present with both neck and shoulder pain, it can present a significant diagnostic dilemma[3]. "Hip spine syndrome" has recently been described, and "neck-shoulder syndrome" likely represents an analogous entity involving the cervical spine and upper limb [4].
It is estimated that among primary care office visits, neck pain accounts for approximately $20-30 \%$ and shoulder pain for $10-20 \%$ of musculoskeletal complaints. From this population, combined neck and shoulder problems account for approximately 6-10\% $[3,5,6,7]$.

\section{Discussion}

Concomitant neck and shoulder pain presentations can be separated into four different categories: Primary neck pathology with referred pain to the shoulder, primary shoulder pathology with referred pain to the neck, primary neck and primary shoulder pathology, and primary neck pathology resulting in secondary shoulder pathology. 


\section{Primary neck pathology with referred pain to the shoulder}

An isolated C5 and/or C6 radiculopathy without shoulder pathology could certainly present with neck and shoulder pain due to C5 and C6 dermatomal symptoms corresponding to the shoulder region. C5 or C6 myotomal pain can cause pain in the deltoid, scapula and biceps, and can mimic shoulder pathology $[8,9]$. This scenario is typically straightforward as the physical examination will be absent of shoulder impingement signs. Classically, cervical radiculopathy examination can demonstrate positive cervical root impingement signs (Spurling's maneuver), myotomal weakness, dermatomal sensory abnormalities and blunted reflexes in a specific root distribution. Several neuropathies involving brachial plexus and its proximal branches will also refer pain to neck and shoulder simultaneously.

\section{Primary shoulder pathology with referred pain to the neck}

Primary shoulder pathology should not directly lead to neck pathology, and such cases are not well described in the literature. Nevertheless, patients with shoulder pathology may develop pain and tightness in the trapezius muscle on the ipsilateral side and referred pain in the cervical area. Restricted motion at the glenohumeral joint may also lead to overuse and pain in the scapulothoracic musculature. A general concern in musculoskeletal medicine is that symptomatic pathology in a joint may refer pain to a joint below and/or above.

\section{Primary neck and primary shoulder pathology}

Degenerative arthritis can affect multiple joints. Thus, many patients may have both glenohumeral arthritis and cervical spondylosis. The radiographic incidence of glenohumeral arthritis is reported as $32.8 \%$ in people over 60 years of age [10]. Radiographic evidence of cervical spondylosis is present in $50 \%$ of people over 50 years of age and $75 \%$ of individuals over 65 years of age [11]. As both conditions are common, both can present as "pain generators."

\section{Primary neck pathology resulting in secondary shoulder pathology}

Primary neck pathology resulting in secondary shoulder pathology is mechanically plausible although not proven. For instance, $\mathrm{C} 5$ and/or C6 cervical radiculopathy can result in rotator cuff, deltoid, biceps and scapular muscle weakness as these nerve roots innervate the shoulder girdle musculature. This could produce muscle imbalance and poor shoulder/scapular mechanics. A patient may present with features of both cervical radiculopathy and shoulder impingement syndrome in this scenario. In clinical practice, it is not uncommon to see a patient with chronic neck pain presenting with insidious onset of shoulder pain later in the course. Authors are proposing this unique presentation be referred to as "neck-shoulder syndrome." Although most clinicians would treat this as separate neck and shoulder pain, they may be related diagnoses.

\section{Literature Search}

We conducted a comprehensive search in the PubMed database in order to identify relevant studies on "neck-shoulder syndrome." Based on the review of the available literature, there are no agreed upon clinical criteria for a diagnosis of "neck-shoulder syndrome" despite its common clinical presentation nor is there a well described "neck-shoulder syndrome." As with any other syndrome, management differences cannot actually be studied until the entity has been appropriately defined. This article will concentrate on how to best approach patients presenting with both neck and shoulder pain while describing features of proposed "neckshoulder syndrome."

\section{Presentation}

In patients presenting with neck and shoulder pain, a thorough history is paramount in identifying the etiology of the patient's pain.

Location: Patients with primary neck pathology can experience pain extending beyond the neck based on the etiology. Disorders that affect the lower cervical nerve roots will often result in pain distal to the shoulder which can be characterized by radiation into the arm in a clear dermatomal or myotomal distribution [12]. In addition, Dwyer et al described reproducible pain patterns that can refer into the shoulder, trapezius and occiput from cervical zygapophyseal joint pathology [13-15]. Pain from a primary shoulder problem can also refer pain to the neck, periscapular region and distally into the arm although not typically extending below the elbow [16]. Associated paresthesias are not classically associated with a primary shoulder problem.

Onset: Onset of symptoms is also a key component of the history. Degenerative cervical pathology can have insidious onset although acute disc herniations can have a sudden onset that may be precipitated by trauma. Whiplash injuries are known to precipitate neck pain which can be of myofascial and/or cervical facet in origin. Shoulder disorders can also be of insidious (overuse injuries) or acute onset (trauma). Sudden onset of shoulder pain with restricted motion can be associated with acute calcific tendinitis or adhesive capsulitis. Neuralgic amyotrophy (Parsonage Turner syndrome/ brachial neuritis) has a unique presentation where patients usually experience severe, acute pain following exercise, recent illness, immunization, surgery or trauma [17]. As the initial severe pain starts to resolve, neurological deficits will become apparent, which is in contrast to most presentations of cervical radiculopathy where pain will continue with associated neurological symptoms. Onset of symptoms plays a key role in proper identification of proposed "neck-shoulder syndrome." Development of shoulder pain (especially in the absence of injury) after onset of neck/radicular pain can be considered primary neck pathology with secondary 
shoulder pathology and can be referred to as "neck-shoulder syndrome." Shoulder pain in this scenario is likely secondary to rotator cuff and periscapular muscle weakness/imbalance caused by $\mathrm{C} 5$ and/or C6 cervical radiculopathy. This clinical scenario is not well studied in the literature, hence prevalence and incidence is not known. Among patients with cervical radiculopathy, studies report a frequency of C5 nerve root involvement at 5-10\%, C6 at 20-25\%, and $\mathrm{C} 7$ at $45-60 \%[12,18]$.

Exacerbating factors: Pain with overhead arm movements generally suggests primary shoulder pathology. However same pattern can also be present in peripheral neuropathies like thoracic outlet syndrome and spinal accessory, suprascapular, or axillary neuropathy. Shoulder pain with side lying on the affected upper limb tends to be associated with shoulder impingement and acromioclavicular joint arthropathy.

Features of systemic diseases: In patients who present with neck and shoulder pain in the absence of trauma, the history will need to include an assessment for widespread involvement that may suggest systemic disease processes like fibromyalgia, polymyalgia rheumatica, myofascial pain syndrome and myopathy.

Red flags: The history should also include an evaluation for findings to suggest a disease process that requires more urgent evaluation. Red-flag symptoms to assess for include gait imbalance, hand clumsiness, bowel/bladder dysfunction (cervical myelopathy), pain after high impact trauma (fractures), unintentional weight loss (Pancoast tumor), chest pain (cardiac ischemia), blurry vision, nausea/vomiting and vertigo (vertebral artery dissection/ insufficiency).

\section{Physical Examination}

In addition to a detailed history, a thorough physical examination is key for proper diagnosis and identification of the pain generator(s). A thorough neurological exam plays an essential role in distinguishing neck from shoulder pathology. Sensory, motor and reflex changes in a specific nerve root distribution are characteristic of cervical radiculopathy. C5 and C6 cervical radiculopathies may result in periscapular and shoulder/rotator cuff muscle weakness while C7 radiculopathy is unlikely to cause shoulder weakness. Rotator cuff pathology may result in shoulder weakness with preserved elbow flexion while a C5 radiculopathy can result in weakness of both. Testing deltoid strength with the arms at the sides instead of in shoulder abduction can aid in differentiating pain inhibition versus true weakness.

\section{Provocative Testing}

Provocative testing can assist in the diagnosis of cervical and shoulder disorders $[9,19]$. Among them, provocative tests for shoulder impingement may help distinguish primary versus secondary shoulder pathology in proposed "neck-shoulder syndrome"(Table 1) [18-30].

In patients with neck and shoulder pain, one test by itself may not have enough sensitivity and specificity to make a diagnosis and most physical exam maneuvers are not pathognomonic. A combination of multiple exam components and a thorough history are necessary to accurately identify the etiology of symptoms.

Table 1: Provocative tests for common cervical and shoulder problems and reported validity.

\begin{tabular}{|c|c|c|}
\hline Diagnosis & Provocative test & Test validity \\
\hline Cervical radiculopathy & Spurling's test & $\begin{array}{l}\text { Sensitivity: } \sim 50 \%{ }^{18,20,21} \\
\text { Specificity: }>80 \%^{18,20,21}\end{array}$ \\
\hline $\begin{array}{c}\text { Subacromial impingement/rotator cuff } \\
\text { pathology }\end{array}$ & Hawkins test & $\begin{array}{l}\text { Sensitivity: } 58-92 \%^{22-24} \\
\text { Specificity: } 25-67 \%^{22-24}\end{array}$ \\
\hline Labral pathology & Obrien's test & $\begin{array}{l}\text { Sensitivity: } 67-100 \%^{25,26} \\
\text { Specificity: } 41-99 \%^{25,26}\end{array}$ \\
\hline Biceps tendonitis & Yerguson's test & $\begin{array}{l}\text { Sensitivity: } 43-66 \%^{27,28} \\
\text { Specificity: } 79-81 \%^{27,28}\end{array}$ \\
\hline Acromioclavicular pathology & Apley scarf test & $\begin{array}{c}\text { Sensitivity: } 67-77 \%^{29} \\
\text { Specificity: } 79 \%^{29}\end{array}$ \\
\hline Glenohumeral instability & Apprehension test & $\begin{array}{l}\text { Sensitivity: } 100 \%^{30} \\
\text { Specificity: } 86 \%^{30}\end{array}$ \\
\hline
\end{tabular}

\section{Diagnostic Testing}

When presented with both neck and shoulder pain, history and physical exam should dictate appropriate use of diagnostic tests. Although imaging studies such as radiography, CT and MRI can reveal many pathologies, further testing should be done to identify the true pain generator. When suspecting pain mediated by a cervical zygapophyseal joint, cervical diagnostic medial branch blocks can be considered. A shoulder injection of lidocaine with or without corticosteroid can be done for diagnostic and perhaps therapeutic reasons. In cases of secondary shoulder pathology, this may give the patient partial benefit, but a primary cervical 
pathology should still be investigated [9]. Electrodiagnostic testing with electromyography (EMG) and nerve conduction studies (NCS) can be employed when suspecting myopathy, brachial plexopathy, peripheral neuropathy or radiculopathy. When evaluating neck and shoulder pain, scientific evidence suggests using a combination of history, physical examination, imaging modalities, diagnostic injections and electrodiagnostic study to make the appropriate diagnosis.

\section{Treatment}

Success of any proposed treatment algorithms will depend on an accurate diagnosis. There is scant evidence on how to approach the concomitant presentation of neck and shoulder pain. Treatment should be geared toward the primary site of pathology whether it be the cervical spine or the shoulder [3]. Lack of current literature evidence underscores the importance of describing a "neckshoulder syndrome," as it can lead to studies looking at management differences. It can be hypothesized that in patients with cervical radiculopathy with secondary rotator cuff impingement, treatment of the primary lesion will likely yield eventual improvement at the secondary site although no studies have been done on this topic.

\section{Conclusion}

The concomitant presentation of shoulder and neck pain is a common scenario in primary care and orthopedic offices and can present a diagnostic and therapeutic dilemma. A careful history and thorough physical examination along with ancillary studies can often yield the correct diagnosis and successful treatment. Primary neck pathology resulting in secondary shoulder pathology is mechanically plausible but not proven. Authors are proposing this scenario to be described as "neck-shoulder syndrome." Appropriately describing a "neck-shoulder syndrome" can lead to further studies looking at management differences. A prospective study looking at incidence of shoulder impingement signs in chronic C5 and/or C6 radiculopathy patients could be helpful in delineating diagnostic criteria for "neck-shoulder syndrome." Above all, it is paramount to take a comprehensive and holistic approach towards patients presenting with concomitant neck and shoulder pain as the symptoms may not always represent isolated entities.

\section{Acknowledgements}

The authors would like to thank Dr. David Janerich for his help with the development of the article.

\section{References}

1. Gorski JM, Schwartz LH (2003) Shoulder impingement presenting as neck pain. J Bone Joint Surg Am 85-A(4): 635-638.

2. Compere EL (1952) The neck, shoulder, and arm syndrome. New Orleans Med Surg J 104(12): 473-477.

3. Prather H, Cheng A, Steger May K, Maheshwari V, Van Dillen L (2018) Association of Hip Radiograph Findings with Pain and Function in Patients Presenting with Low Back Pain. PMR 10(1): 11-18.
4. Manifold SG, McCann PD (1999) Cervical radiculitis and shoulder disorders. Clin Orthop Relat Res 368: 105-113.

5. Manchikanti L, Nampiaparampil DE, Candido KD, Bakshi S, Grider JS, et al. (2015) Do cervical epidural injections provide long-term relief in neck and upper extremity pain? A systematic review. Pain Physician18(1): $39-60$.

6. Cassou B, Derriennic F, Monfort C, Norton J, Touranchet A (2002) Chronic neck and shoulder pain, age, and working conditions: longitudinal results from a large random sample in France. Occup Environ Med 59(8): 537-544

7. Rekola KE, Levoska S, Takala J, Keinanen-Kiukaanniemi S (1997) Patients with neck and shoulder complaints and multisite musculoskeletal symptoms a prospective study. J Rheumatol 24(12): 2424-2428.

8. Caridi JM, Pumberger M, Hughes AP (2011) Cervical radiculopathy: a review. HSS J 7(3): 265-272.

9. Fish DE, Gerstman BA, Lin V (2011) Evaluation of the patient with neck versus shoulder pain. Phys Med Rehabil Clin N Am 22(3): 395-410.

10. Kerr R, Resnick D, Pineda C, Haghighi P (1985) Osteoarthritis of the glenohumeral joint: a radiologic-pathologic study. AJR Am J Roentgenol 144(5): 967-972.

11. Brooker AE, Barter RW (1965) Cervical spondylosis. A clinical study with comparative radiology. Brain 88(5): 925-936.

12. Cohen SP (2015) Epidemiology, diagnosis, and treatment of neck pain. Mayo Clin Proc 90(2): 284-299.

13. Fukui S, Ohseto K, Shiotani M, Ohno K, Karasawa H (1996) Referred pain distribution of the cervical zygapophyseal joints and cervical dorsal rami. Pain 68(1): 79-83.

14. Dwyer A, Aprill C, Bogduk N (1990) Cervical zygapophyseal joint pain patterns. I: A study in normal volunteers. Spine 15(6): 453-457.

15. Aprill C, Dwyer A, Bogduk N (1990) Cervical zygapophyseal joint pain patterns. II: A clinical evaluation. Spine 15(6): 458-461.

16. Kennedy DJ, Mattie R, Nguyen Q Hamilton S, Conrad B (2015) Glenohumeral Joint Pain Referral Patterns: A Descriptive Study. Pain Med 16(8): 1603-1609.

17. Suarez GA, Giannini C, Bosch EP, Barohn RJ, Wodak J (1996) Immune brachial plexus neuropathy: suggestive evidence for an inflammatoryimmune pathogenesis. Neurology 46(2): 559-561.

18. Radhakrishnan K, Litchy WJ, O Fallon WM, Kurland LT (1994) Epidemiology of cervical radiculopathy. A population-based study from Rochester, Minnesota, 1976 through 1990. Brain 117 (Pt 2): 325-335.

19. Gismervik SO, Drogset JO, Granviken F, Ro M, Leivseth G (2017) Physical examination tests of the shoulder: a systematic review and meta-analysis of diagnostic test performance. BMC Musculoskelet Disord 18(1): 41.

20. Rubinstein SM, Pool JJ, van Tulder MW, Riphagen, II (2007) A systematic review of the diagnostic accuracy of provocative tests of the neck for diagnosing cervical radiculopathy. Eur Spine J 16(3): 307-319.

21. Viikari Juntura E, Porras M, Laasonen EM (1989) Validity of clinical tests in the diagnosis of root compression in cervical disc disease. Spine 14(3): 253-257.

22. MacDonald PB, Clark P, Sutherland K (2000) An analysis of the diagnostic accuracy of the Hawkins and Neer subacromial impingement signs. J Shoulder Elbow Surg 9(4): 299-301.

23. Calis M, Akgun K, Birtane M, Karacan I, Calis H (2000) Diagnostic values of clinical diagnostic tests in subacromial impingement syndrome. Ann Rheum Dis 59(1): 44-47.

24. Park HB, Yokota A, Gill HS, El Rassi G, McFarland EG (2005) Diagnostic accuracy of clinical tests for the different degrees of subacromial impingement syndrome. J Bone Joint Surg Am 87(7): 1446-1455. 
25. Stetson WB, Templin K (2002) The crank test, the O'Brien test, and routine magnetic resonance imaging scans in the diagnosis of labral tears. Am J Sports Med 30(6): 806-809.

26. Burkhart SS, Morgan CD, Kibler WB (2000) Shoulder injuries in overhead athletes. The "dead arm" revisited. Clin Sports Med 19(1): 125-158.

27. Lasbleiz S, Quintero N, Ea K, Petrover D, Aout M (2014) Diagnostic value of clinical tests for degenerative rotator cuff disease in medical practice. Ann Phys Rehabil Med 57(4): 228-243.
28. Holtby R, Razmjou H (2004) Accuracy of the Speed's and Yergason's tests in detecting biceps pathology and SLAP lesions: comparison with arthroscopic findings. Arthroscopy 20(3): 231-236.

29. Chronopoulos E, Kim TK, Park HB, Ashenbrenner D, McFarland EG (2004) Diagnostic value of physical tests for isolated chronic acromioclavicular lesions. Am J Sports Med 32(3): 655-661.

30. Bushnell BD, Creighton RA, Herring MM (2008) The bony apprehension test for instability of the shoulder: a prospective pilot analysis. Arthroscopy 24(9): 974-982

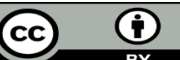

This work is licensed under Creative Commons Attribution 4.0 License

To Submit Your Article Click Here:

Submit Article

DOI: $10.32474 /$ GJAPM.2019.01.000103

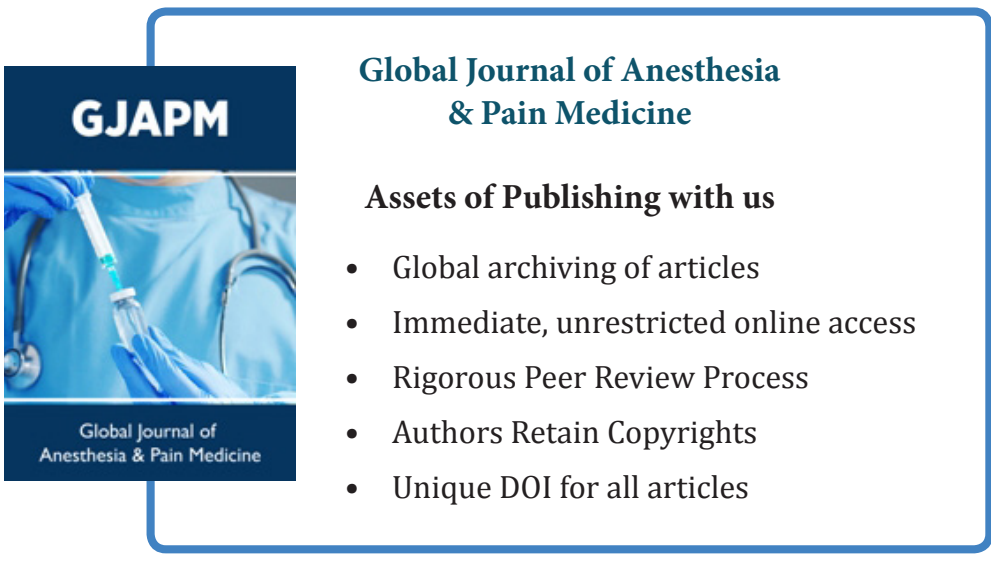

